Research Article

\title{
A Multipath Processing Technology Based on Multiparameter-Combined Observation in GNSS
}

\author{
T. Y. Zhou ${ }^{1,2}$ and B. W. Lian (iD) \\ ${ }^{1}$ School of Electronics and Information, Northwestern Polytechnical University, Xi'an 710129, China \\ ${ }^{2}$ School of Information, Hunan University of Humanities Science and Technology, Loudi 417000, China \\ Correspondence should be addressed to B. W. Lian; bwlian@126.com
}

Received 25 February 2021; Revised 8 April 2021; Accepted 29 April 2021; Published 17 May 2021

Academic Editor: Chi-Hua Chen

Copyright (c) 2021 T. Y. Zhou and B. W. Lian. This is an open access article distributed under the Creative Commons Attribution License, which permits unrestricted use, distribution, and reproduction in any medium, provided the original work is properly cited.

\begin{abstract}
In the Global Navigation Satellite System (GNSS), the multipath error affected by many aspects is the main error source that affects satellite navigation, and it is difficult to establish a much more accurate model to analyze it. Based on the ground multipath reflection model, it firstly deeply studies the influence of GNSS satellite orbit parameters on multipath fading frequency and establishes a multipath signal model related to satellite orbit parameters. Secondly, the influence of carrier phase cycle slip, receiver clock adjustment, and GNSS satellite orbit on multiparameter- (MP-) combined observations is analyzed in detail based on the measured data. Finally, aiming at the common phenomenon of code-carrier divergence in the Beidou system, the elevation-based pseudorange correction model and a sidereal filtering are built to correct the MP errors; experiments with measured data show that there is a fluctuation range reduction of $35.7 \%$ after sidereal filtering when the receiver reaches a steady state.
\end{abstract}

\section{Introduction}

The satellite navigation system has become one of the most important technical means in modern navigation and positioning measurement $[1,2]$. To pursue more accurate positioning results, it is necessary to analyze the reasons that affect the accuracy of a satellite navigation system and take targeted measures to reduce and correct the relevant errors and obtain a better positioning performance $[3,4]$. There are many factors which will affect the positioning error of satellite navigation, among which ionospheric error and tropospheric error can be eliminated by differential technique or some mathematical methods $[5,6]$. However, the multipath error is affected by many aspects, and it is difficult to establish a more accurate model for it and eliminate it by the general method, so multipath error has become the main error source that affects satellite navigation $[7,8]$. As early as the GPS demonstration test, the influence of the multipath effect on satellite ranging performance and positioning results was considered by the designers. The mathematical relationship between the multipath effect and pseudorandom code tracking derived by
Hagerman in 1973 is the basis of multipath effect analysis. In the late 1970s, the researchers tested the multipath effect and its effect on the receiver $[9,10]$. The influence of the multipath effect on short baseline carrier phase differential positioning was studied by MIT and Draper laboratories in the early 1980s. For GPS systems, the effect of the multipath effect on carrier phase differential positioning can be reduced to several centimeters by averaging the observation results greater than 1 hour $[11,12]$. Since then, with the deepening of the research, the research on multipath effect in the field of satellite positioning and navigation mainly focuses on the environment and channel modelling, multipath measurement technology, antimultipath antenna, anti-multipath receiver digital signal processing technology, anti-multipath data postprocessing method, and multipath research under the new signal system and evaluation of anti-multipath performance. Based on the traditional antenna design, an antenna technology with a standing wave ratio below $1.5 \mathrm{~m}$ is studied by using $L$ feed and double-layer structure in [4]. A technique of adding parasitic circular metal patch as the top-loaded antenna is studied based on a prefeed network in [11]. 


\section{Literature Review}

Multipath is the main systematic error of the GNSS short baseline positioning. Multipath error cannot be eliminated by the double-differenced technique and is difficult to parameterize, which severely restrict the high-precision GNSS positioning application. A correlation reconstruction algorithm for energy compensation under the BOC system is studied in [13]. A time-division multiplexing dual reference waveform multipath suppression algorithm for TMBOC signals is studied in [14]. Based on Bayesian estimation, a multipath estimation technique of the particle filter algorithm is studied in [15]. Based on maximum likelihood estimation, an off-line mesh search and online iteration method is studied in [16] to suppress short multipath delay. In [17], the authors propose a new multipath mitigation strategy in the coordinate domain that shakes off the formation mechanism of multipath; a convolutional neural network (CNN) long short-term memory (LSTM) method is used to mine the deep multipath features in GNSS coordinate series. An advanced receiver autonomous integrity monitoring (ARAIM) method is proposed to evaluate GNSS integrity in [18], which mainly focuses on validating whether the multipath error model in the ARAIM algorithm is conservative enough for GEO satellites. In [19], the authors address the problem of GNSS multipath mitigation using antenna arrays, in which a new data-dependent beamforming technique is proposed, which is based on the wellknown Capon beamformer. This technique aims to avoid the typical cancellation phenomenon between signal and correlated multipaths, by exploiting the known power of the direct signal at the receiver. To this effect, a measure of the correlation between the signal and multipaths is obtained in the matrix form, and it is then subtracted from the spatial correlation matrix of the received signal. This results in a new spatial correlation matrix that is used for the final Power-Based Capon beamformer. The behaviour of this technique is justified mathematically, and it is supported by several numerical results.

Here, this paper establishes a much more accurate multipath signal model, and the basic ideas can be concluded as follows: firstly, the influences of GNSS satellite orbit parameters on multipath fading frequency are studied and analyzed, and a multipath fading frequency model related to satellite orbit parameters is established. Secondly, the MPcombined observations are extracted by the code subtraction carrier phase, and the influences of carrier phase cycle slip, receiver clock correction, and GNSS satellite orbit to MP observations are analyzed by measured data. Finally, the elevation correlation pseudorange correction model and sidereal filtering are used to correct the common codecarrier divergence phenomenon in the Beidou system. The rest of the paper is organized as follows. In Section 3, the mathematical model of multipath signals is established. In Section 4, the MP-combined observations are analyzed. In Section 5, the suppression of MP signals is discussed. Finally, in Section 6, we conclude our work.

\section{Mathematical Model of the Multipath Signal}

3.1. Fading Frequency of the Multipath Signal. Considering generality, assume that the satellite signal reaches the receiver at the same time as the reflected signal, and the received synthetic signal $s(t)$ can be described as

$$
s(t)=p(t) \sin \left(\omega_{0} t\right)+|\Gamma| p(t-\Delta t) \sin \left(\omega_{0} t+\Delta \phi_{m}(t)\right),
$$

where $p(t)$ is the signal amplitude (or spread spectrum code or message), $|\Gamma|$ is the attenuation coefficient, $\Delta t$ is the multipath delay $(s), \omega_{0}$ is the angular frequency of the direct signal and contains the influence of the doppler shift $\Delta \omega_{0}=2 \pi \Delta f_{0}, \Delta \phi_{m}(t)=\Delta \varphi_{m}+\left(\Delta w_{m}-\Delta w_{0}\right) t$ is the phase difference between the reflected signal and the direct signal, where $\Delta \varphi_{m}$ is the initial phase of the reflected signal, $\left(\Delta w_{m}-\Delta w_{0}\right)$ is the Doppler frequency difference between the reflected signal and the direct signal, and $|\Gamma|, \Delta t$, and $\Delta \phi_{m}(t)$ describe the basic characteristics of the synthesized signal.

Due to the relative motion between the antenna of the ground receiver and the satellite, the multipath delay $\Delta t$ and phase difference $\Delta \phi_{m}(t)$ will change randomly with time, which will cause the change of the carrier frequency of the reflected wave and produce an increment $\Delta f_{m}(t)$ on the carrier nominal frequency, which is called multipath fading frequency. According to the relationship between the frequency and phase of the sinusoidal signal, $\Delta f_{m}(t)$ can be expressed in [10]:

$$
\Delta f_{m}(t)=\frac{1}{2 \pi} \frac{\mathrm{d} \Delta \phi_{m}(t)}{\mathrm{d} t}=\frac{1}{2 \pi} \frac{\mathrm{d}\left[\Delta \varphi_{m}+\left(\Delta w_{m}-\Delta w_{0}\right) t\right]}{\mathrm{d} t} .
$$

It can be seen from equation (2) that $\Delta f_{m}(t)$ depends on the Doppler frequency difference $\left(\Delta w_{m}-\Delta w_{0}\right)$; when it changes rapidly with time, the relative speed between the ground receiving antenna and the satellite will be relatively fast, and $\Delta f_{m}(t)$ will be larger.

3.2. Multipath Reflection Model. The multipath reflection model (see Figure 1), in which $\theta^{s}(t)$ is the elevation angle of the

satellite at $t$ time in the Earth-centered Earth-fixed (ECEF) coordinate system. $A$ is the phase center of the user receiver, whose height from the ground is $h, R$ is the incident point of the reflected signal, and $L_{\mathrm{LOS}}(t)$ and $L_{\mathrm{NLOS}}(t)$ represent the propagation path length of the direct signal and reflected signal, respectively.

Since the satellite is far from the satellite, it is generally considered that the direct signal is parallel to the reflected signal, and the vertical line of the direct signal and the reflected signal is made at $R$; with the vertical foot is $D$, the multipath geometric distance delay of the reflected signal relative to the direct signal $L(t)$ can be expressed as follows: 


$$
L(t)=\left|L_{\mathrm{NLOS}}(t)-L_{\mathrm{LOS}}(t)\right|=|A R|\left(1-\cos 2 \theta^{s}(t)\right)=\frac{h}{\sin \theta^{s}(t)} \cdot 2 \sin ^{2} \theta^{s}(t)=2 h \cdot \sin \theta^{s}(t)
$$

According to equation (3), $L(t)$ is a function of antenna height $h$ and satellite elevation angle $\theta^{s}(t)$. According to the theory of electromagnetic wave propagation, the relative phase of multipath $\Delta \phi_{m}(t)$ is explained in [11]:

$$
\Delta \phi_{m}(t)=\frac{2 \pi L(t)}{\lambda}=\frac{4 \pi h \cdot \sin \theta^{s}(t)}{\lambda},
$$

where $\lambda$ is the signal wavelength.

Combine equations (4) with (2), the multipath fading frequency $\Delta f_{m}(t)$ can be rewritten as

$$
\Delta f_{m}(t)=\frac{1}{2 \pi} \frac{\mathrm{d} \Delta \phi_{m}(t)}{\mathrm{d} t}=\frac{2 h}{\lambda} \cdot \frac{\mathrm{d}\left[\sin \theta^{s}(t)\right]}{\mathrm{d} t} .
$$

3.3. Satellite Orbital Parameters. In ECEF coordinates, the geometric relationship between the satellite orbits and Earth (see

Figure 2, in which, $R_{e}$ is the radius of the Earth whose center is $O$, and the GNSS receiver antenna is placed at $A$, a point on the ground (which can fall at any point in the sphere; for easy drawing, it is placed at the north pole, the vector $O A$ point to zenith). $P$ is the level plane where the user $A$ is, $S$ represents the spatial position of the satellite relative to the Earth at $t$ time, whose projection point on the horizontal $P$ is $D, r(t)=\|A S\|$ is the line of sight distance between the user and the satellite, $R_{s}=\|O S\|$ represents the geometric distance between the geocentric center $O$ and the satellite, and the satellite elevation angle $\theta^{s}(t)$ is the angle between $\overrightarrow{A S}$ and $A D$ at time $t$.

According to the geometric relation shown in Figure 2 and the cosine theorem, it can be obtained that

$$
\sin \theta^{s}(t)=\frac{R_{s}^{2}-R_{e}^{2}-r^{2}(t)}{2 R_{e} \cdot r(t)} .
$$

Combine (5) with (6), then $\Delta f_{m}(t)$ can be represented as

$$
\Delta f_{m}(t)=-\frac{2 h}{\lambda}\left(\frac{1}{R_{e}}+\frac{\sin \theta^{s}(t)}{r(t)}\right) r^{\prime}(t)
$$

where $h, \lambda, R_{e}, \theta^{s}(t)$, and $r(t)$ can be regarded as known, that is, $\Delta f_{m}(t)$ mainly depends on the unknown $r^{\prime}(t)$, which can be understood as the relative velocity in the direction of the signal propagation.

Let $K=-(2 h / \lambda)\left(\left(1 / R_{e}\right)+\left(\sin \theta^{s}(t) / r(t)\right)\right)$, equation (7) can be simplified as

$$
\Delta f_{m}(t)=K r^{\prime}(t) .
$$

For a ground static receiver, let $\vec{v}$ represent the satellite velocity and $\vec{I}$ represent the unit observation vector, then $r^{\prime}(t)_{s}$ can be regarded as the projection of the satellite velocity on $\vec{I}$, that is [10],

$$
r^{\prime}(t)=v^{s} \cdot \cos \beta
$$

where $\beta$ is the angle between $\vec{v}$ and $\vec{I}$ and satisfies

$$
\cos \beta=\frac{e \sin f^{s}}{\sqrt{1+e^{2}+2 e \cos f^{s}}} \cos \gamma
$$

where $f^{s}$ is the true near point angle of the satellite and $\gamma$ is the angle between the satellite observation vector and geocentric distance vector.

Based on the transformation theory of the coordinate system, $v^{s}$ can be expressed as

$$
v^{s}=\frac{R_{s}}{\sqrt{2}} V,
$$

where

$$
V=\sqrt{\left(w_{e}^{2}+w^{s 2}\right)\left(1+\cos ^{2} \alpha\right)-4 w_{e} w^{s} \cos ^{2} \alpha+\left[w_{e}^{2} \cos 2\left(w^{s} t+w\right)+w^{s 2}\right] \sin ^{2} \alpha}
$$

where $\alpha$ is the inclination angle of the satellite's orbit, $w_{e}$ is the rate of the Earth's self-rotation, $w$ is the distance of the orbit near the Earth's angle, and $w^{s}$ is the angular rate of the satellite.

For the convenience of analysis, we define a Kepler multipath fading (Kepler Multipath Fading, KMPF) factor $\kappa(t)$ :

$$
\kappa(t)=\frac{e \sin f^{s}}{\sqrt{1+e^{2}+2 e \cos f^{s}}} V \cos \alpha .
$$

Combine equations (8)-(10)and (13), we can conclude

$$
\Delta f_{m}(t)=K \kappa(t) R_{s} \cos \gamma \cos ^{-1} \alpha .
$$

\section{MP-Combined Observations}

4.1. Extraction of MP. GNSS pseudorange and carrier phase observation equations can be expressed, respectively, as [12] 


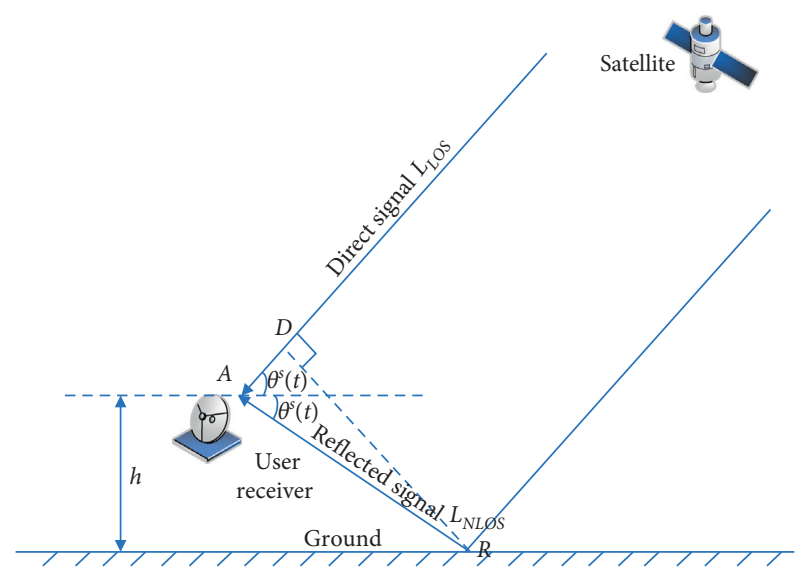

Figure 1: A multipath reflection model.

$$
P=\rho+c\left(\mathrm{~d} t_{r}-\mathrm{d} T^{s}\right)+c\left(T+I+M_{P}+v_{P}+H W\right)+U R E+\varepsilon_{P},
$$

$$
\begin{aligned}
\Phi=\lambda f= & \rho+c\left(\mathrm{~d} t_{r}-\mathrm{d} T^{s}\right)+c\left(T-I+M_{\phi}+v_{\phi}+H W\right) \\
& +U R E+\varepsilon_{\phi}+\lambda N,
\end{aligned}
$$

where $M_{p}$ and $M_{\phi}$ are the specular reflection of pseudorange and carrier phase, respectively, $v_{P}$ and $v_{\phi}$ are the diffuse reflection of pseudorange and carrier phase, respectively, $H W$ is the time delay of the processor, and $U R E$ is the deviation of the satellite orbit.

Ignore the carrier phase errors $\varepsilon_{\phi}, v_{\phi}$, and $M_{\phi}$ with very small values; the difference between pseudorange and carrier phase observation at the same frequency, also named as multiparameter(MP)-combined observation, can be obtained as follows:

$$
(P-\Phi)_{\mathrm{adj}}=c\left(2 I+v_{P}+M_{P}\right)+\varepsilon_{P}-\lambda N,
$$

where the ionospheric delay $2 c I$ can be modified with a double-frequency ionospheric correction method described in [13].

The carrier multipath error can be ignored, for its maximum multipath error is much smaller than that of code multipath error [14]. Hence, the MP-combined observation is usually used to describe the multipath signal. According to the method mentioned above, the modified $M P_{f_{i}}$ of $f_{i}$ frequency with dual-frequency ionospheric method can be described as

$$
M P_{f_{i}}=\left(P_{f_{i}}-\phi_{f_{i}}\right)_{\mathrm{adj}}-2 c I_{f_{i}}=\varepsilon_{P}+c\left(v_{P}+M_{P}\right)-\lambda N .
$$

Since the transformation factor between time and distance is a constant $c$, there is no distinction between the two different physical quantities representing time and distance without confusion in the GNSS positioning field. Therefore, equation (18) can be further simplified as follows:

$$
M P_{f_{i}}=\left(P_{f_{i}}-\phi_{f_{i}}\right)_{\mathrm{adj}}-2 c I_{f_{i}}=\varepsilon_{P}+v_{P}+M_{P}-\lambda N .
$$

4.2. Effect of Carrier Phase Cycle Slip on MP Observations. If there are cycle slips in the carrier phase of the observations, the calculated carrier phase ionospheric delay is inaccurate, which will, in turn, affect the MP value; in particular, a continuous small cycle slip will cause fluctuations in the MP values. Hence, it is necessary to analyze and detect the influence of the carrier phase slip on MP values. The dual-frequency carrier phase method and ionospheric residual method are generally used to detect the cycle slip [15]. Here, the double-frequency pseudorange observations are used for cycle slip detection.

For the convenience of analysis, the smaller error term in the phase measurement equation is ignored, and the carrier phase measurement equation described in equation (16) can be simplified as follows:

$$
\phi=\rho+c\left(\delta t_{u}-\delta t^{u}\right)+c(T-I)+\lambda N .
$$

The phase observations of two adjacent epochs at times $t$ and $t+1$ are calculated; most of the ionospheric and tropospheric delays can be eliminated without the carrier phase slip [16], and the carrier phase difference $\Delta \phi$ can be expressed as

$$
\Delta \phi=\phi^{t+1}-\phi^{t}=\Delta \rho+c\left[\left(\delta t_{u}^{t+1}-\delta t^{u(t+1)}\right)-\left(\delta t_{u}^{t}-\delta t^{u(t)}\right)\right] .
$$

The two-frequency phase observations for GPS $L 1$ and L2 satisfies

$$
\Delta \phi_{L 1} \lambda_{1}-\Delta \phi_{L 2} \lambda_{2}=0
$$

Let

$$
v=\Delta \phi_{L 1}-\frac{\lambda_{2}}{\lambda_{1}} \Delta \phi_{L 2} .
$$

Technically, the cycle slip search of MP observations can be carried out in two steps: firstly, the severe cycle slips and signal tracking interruption are detected with a threshold of $5 \sigma$ or above [20], where $\sigma$ is the mean square error (MSE) of carrier phase difference, marked in the output results and repaired in sections. According to the all-day data collected from Beidou on January 3rd in 2018, the cycle slips and their influences on MP of SV5 B1 are shown in Figure 3.

In Figure 3, the $X$ axis represents epoch time (s) or samples and (a) and (b) show the first difference of ionospheric residuals before and after cycle slip repair, respectively, whose $Y$ axis represents the ionospheric residual (m). The red horizontal line in (a) is the severe cycle slip search threshold, while the blue pulse is the severe cycle slip and tracking interruption that needs to be repaired. The green horizontal line in (b) is the position where the small cycle slips appear. The threshold is chosen according to [21]. (c) is the ionospheric residual curve, in which the $Y$ axis represents the ionospheric residual value, the red asterisk is the location of the severe cycle slip and tracking interrupt, and the green circle is the location where the small cycle slips occur. (d) is the final MP observations, where the blue and red curves correspond to $B 1$ and $B 2$ frequencies, respectively. 


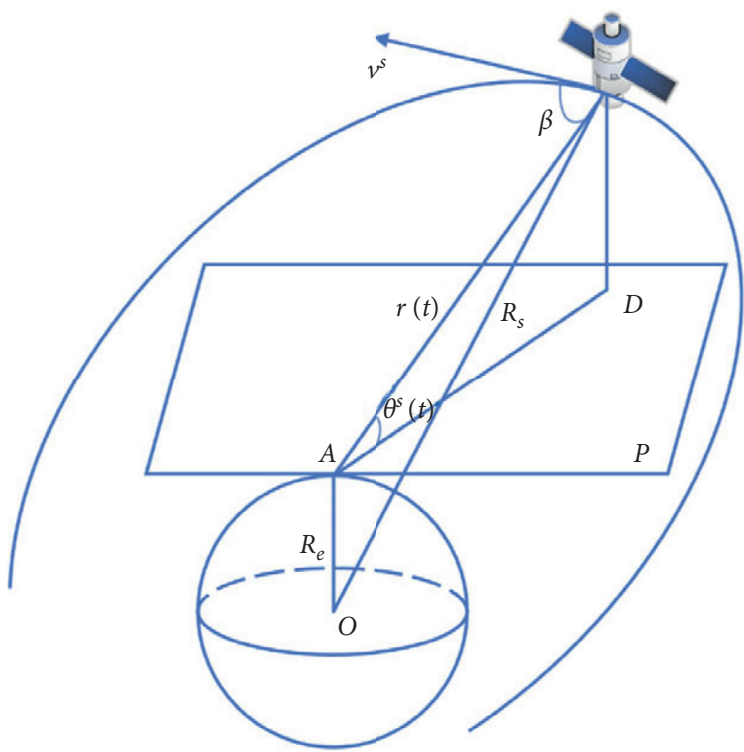

(a)

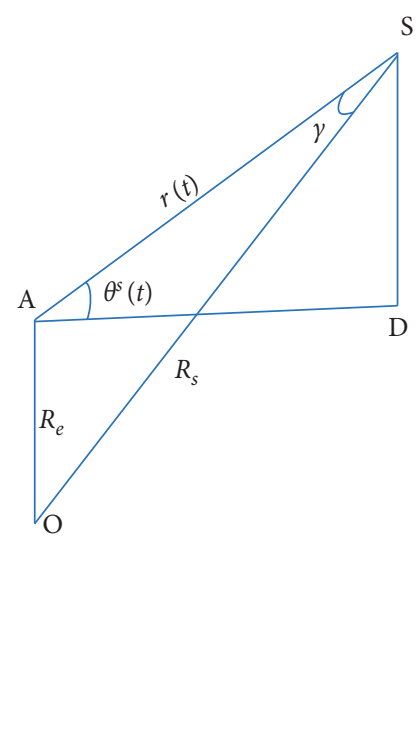

(b)

FIgURE 2: Geometric relationship between the Earth and the satellite orbits. (a) 3D model. (b) Local Magnification Diagram.

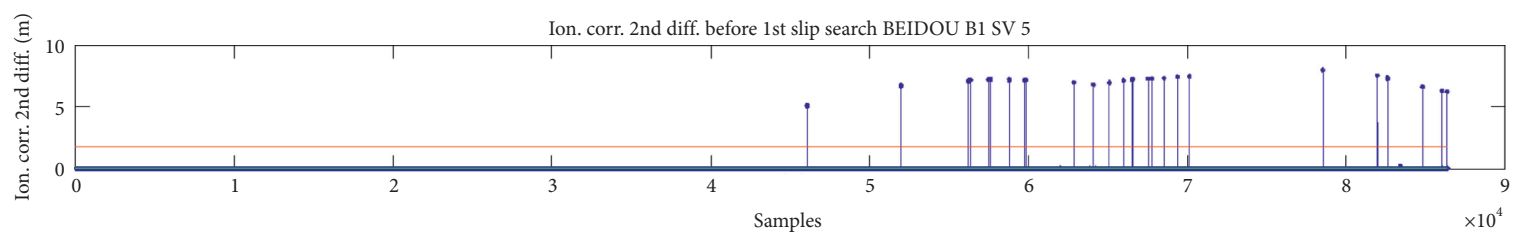

(a)

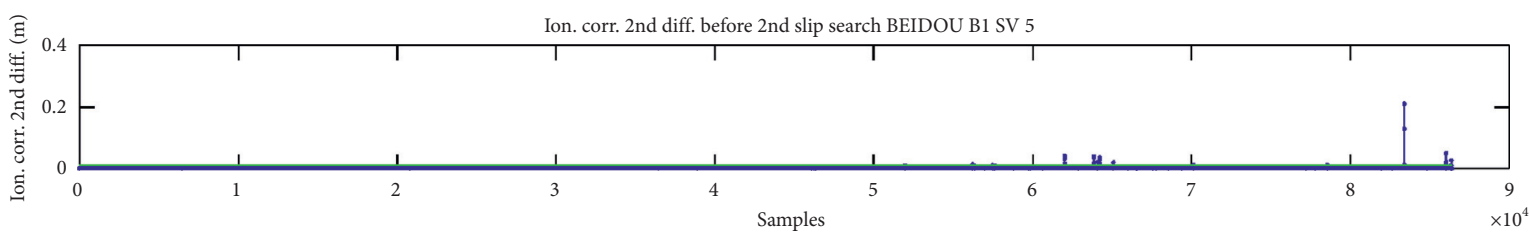

(b)

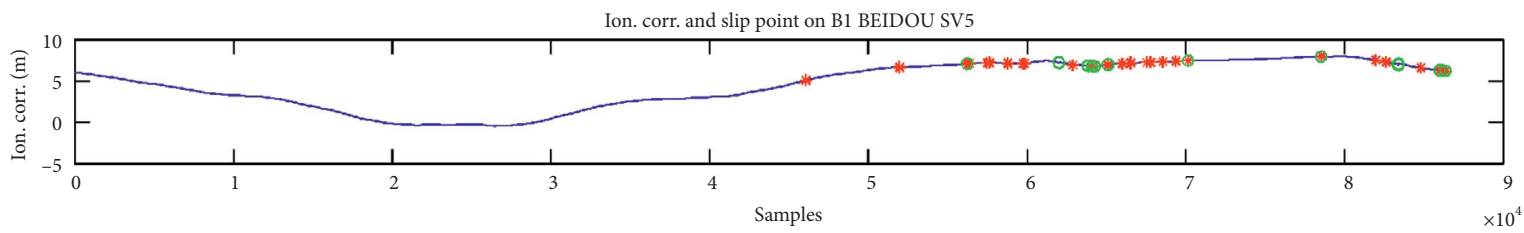

(c)

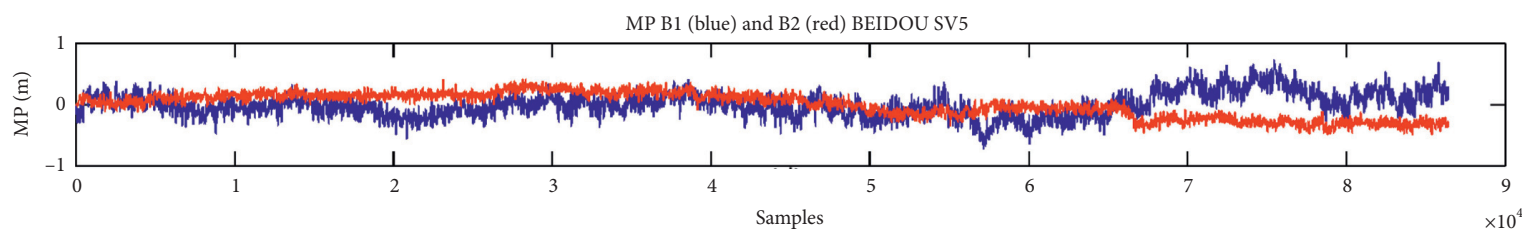

(d)

Figure 3: Effect of the carrier phase cycle slip on MP observations.

4.3. Effect of Receiver Clock Adjustment on MP Observations. There are usually two methods for the receiver to deal with the local clock [21]: one is to use an interval clock adjustment when the cumulative error reaches a certain threshold (such as $0.5 \mathrm{~ms}$ ), the local clock is set back or forward twice the threshold (1 ms), and the other is to use a continuous clock 


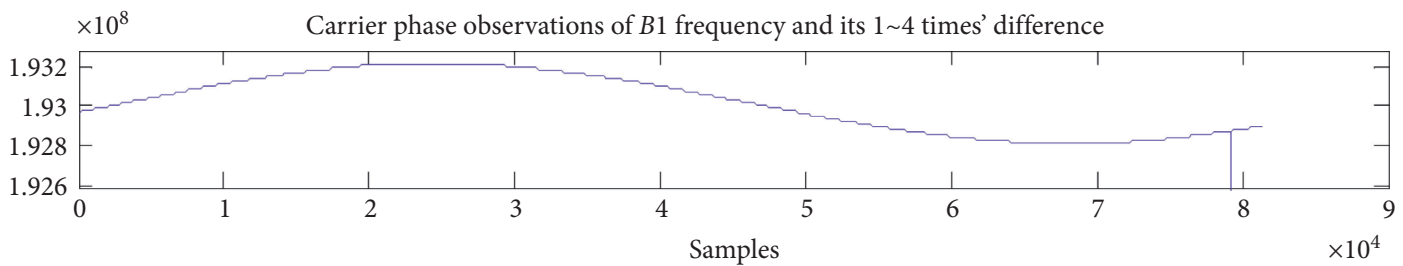

(a)

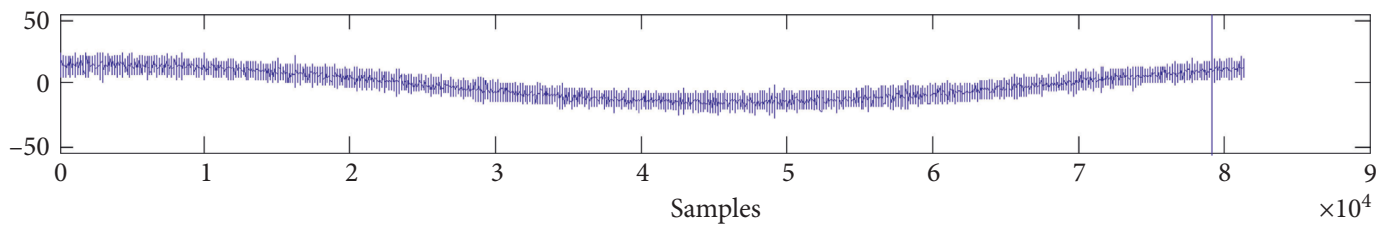

(b)

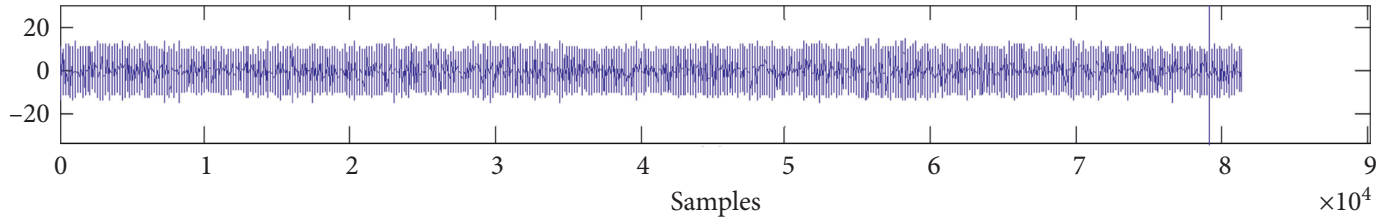

(c)

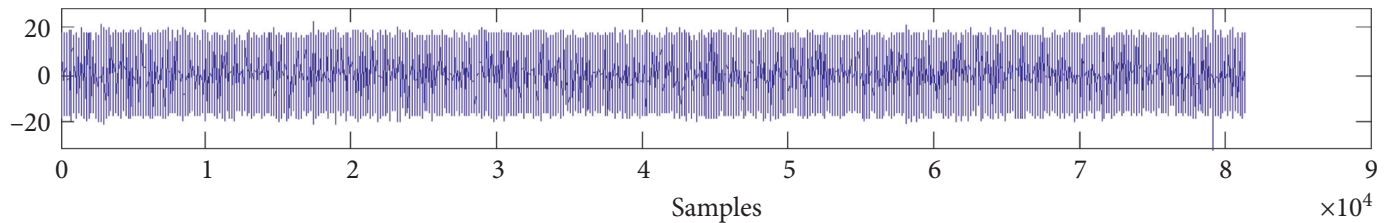

(d)

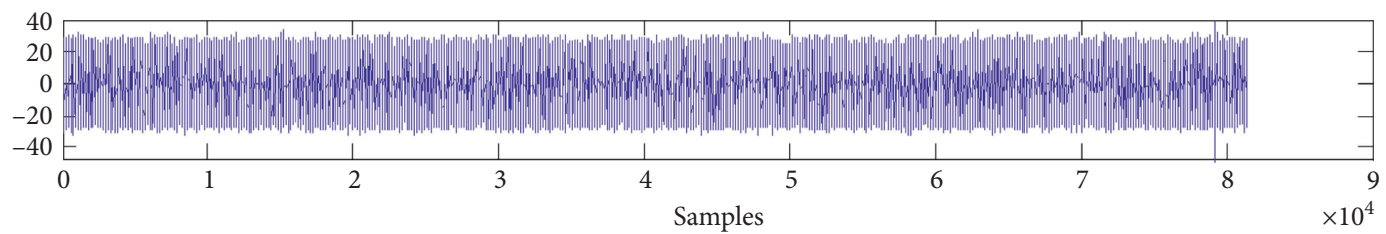

(e)

FIgURE 4: The all-day carrier phase observations of $B 1$ frequency.

adjustment, which adjusts the local clock immediately after the completion of positioning, keeping synchronization with the satellite. The effects of clock adjustment on MP observations in the Aerospace Star receiver are shown in Figures 4 and 5 .

In Figure 4, $X$-axis represents epoch time (s) or samples, $Y$-axis represents the carrier phase observations of $B 1$ frequency $(\mathrm{m})$ and (a)-(e) are the carrier phase observations of $B 1$ frequency and its 1-4 times' difference, respectively.

In Figure 5, the $X$-axis represents epoch time (s) or samples, (a) and (b), $Y$-axis represents the ionospheric residual $(\mathrm{m})$, which is the first difference of ionospheric residuals before and after the cycle slip repairment, respectively, where the red and green horizontal lines are the cycle slip detection thresholds. (c) is the ionospheric residual curve, where the red mark indicates the location of the cycle slip, and (d) is the final MP observations. It can be seen from Figures 4 and 5 that there is no serious cycle slip with the ionospheric residuals, and the high order difference results of the carrier phase are stable within \pm 20 cycles.

\subsection{MP Observations of IGSO/MEO/GEO Satellites}

4.4.1. MP Observations of IGSO/MEO Satellites. There exists code and carrier divergence in the MP observations of Beidou IGSO/MEO satellites which is related to the elevation angle. In the process of satellite transits, the MP decreases gradually with the increase of the elevation angle, and conversely, the MP value increases with the falling of the elevation angle [21]. According to the MP measurement theory, the reason for the decrease of the MP is that the pseudorange measurements are small or the carrier phase measurements are too large. The all-day MP observations of Beidou IGSO (PRN6 10) and MEO (PRN11/14) satellites collected with the Zhongda receiver at the self-built station of Northwest Polytechnic University on June 7th in 2015 are shown in Figure 6.

The MP observations of SV193 L1 frequency and Beidou PRN6 B1 frequency of Quasi-Zenith Satellite System (QZSS) observed at IGS JFNG and IGS XMIS stations on January 3rd in 2016 are shown in Figure 7. 


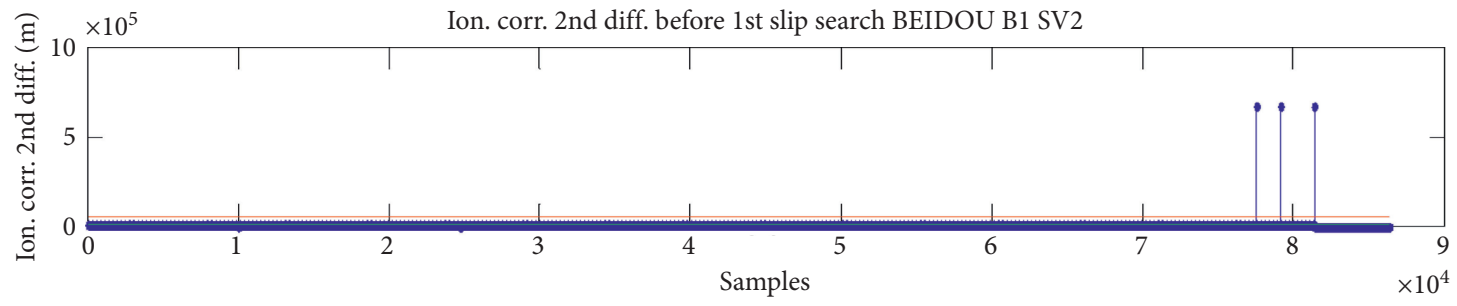

(a)

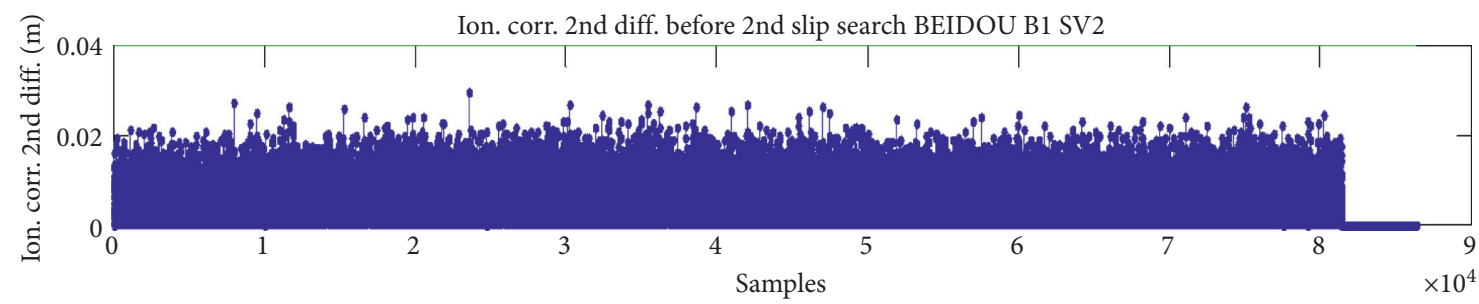

(b)

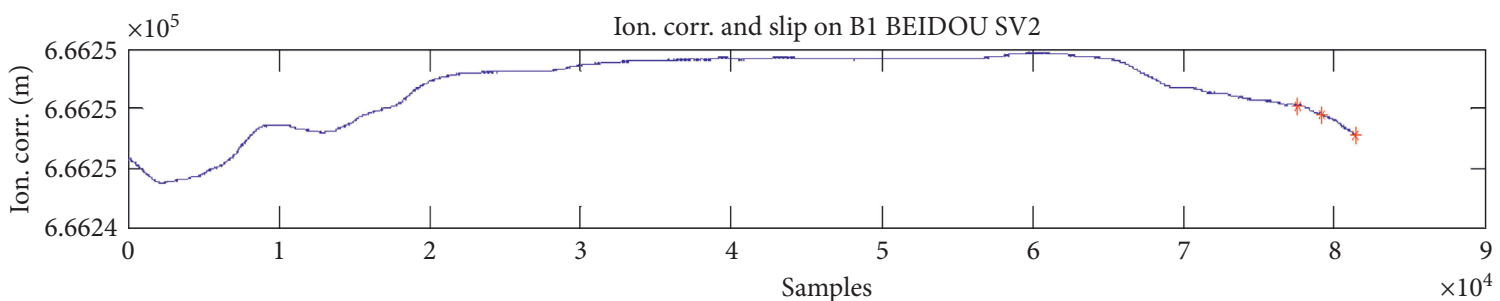

(c)

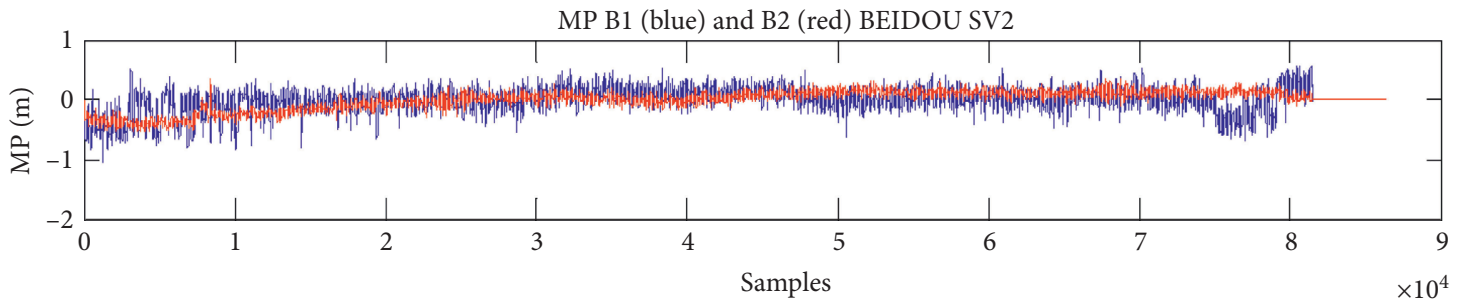

(d)

FIgURE 5: The ionospheric residuals and MP observations before and after cycle slip repairment.

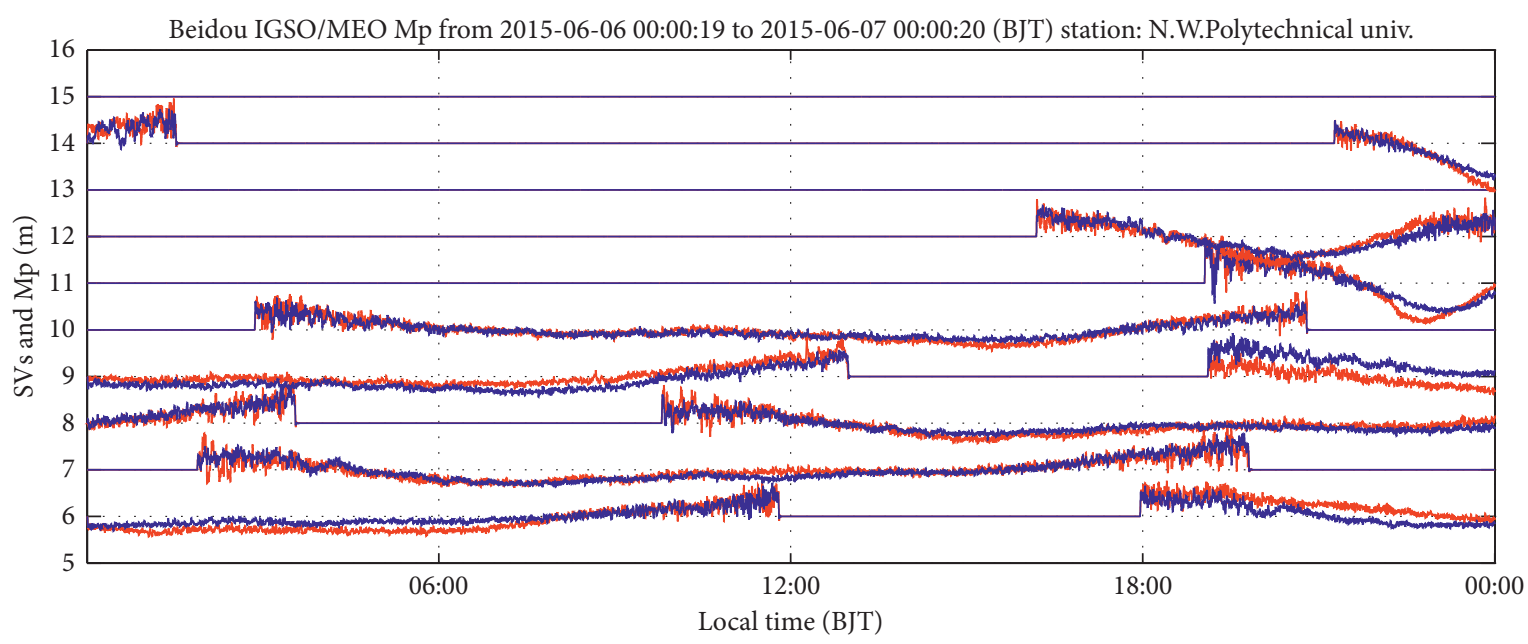

FIgURE 6: MP observations of Beidou IGSO/MEO satellites (red: B2 frequency; blue: $B 1$ frequency). 

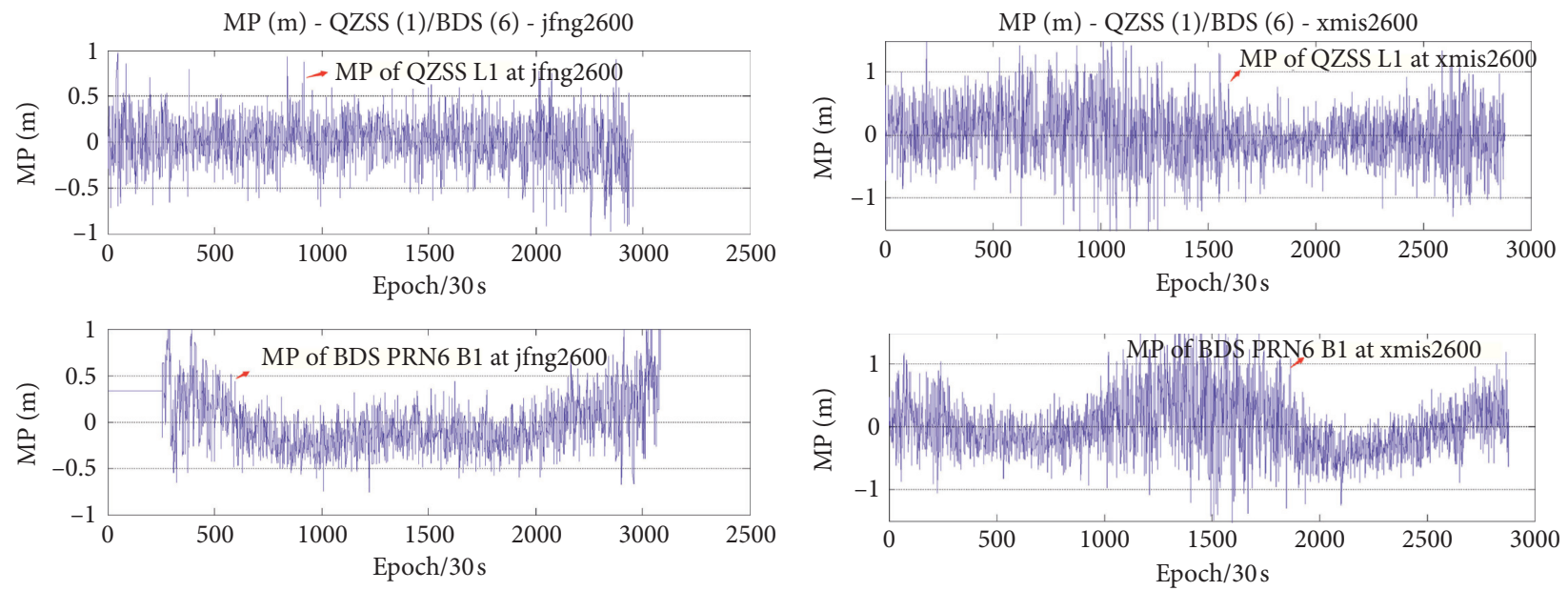

(a)

(b)

FIgure 7: MP observations of Beidou PRN6 and QZSS. (a) MP observations of QZSS L1 and Beidou PRN6 B1 at IGS JFNG station. (b) MP observations of QZSS L1 and Beidou PRN6 B1 at IGS XMIS station.

All the data in Figures 6 and 7 are collected with the same receiver. The analysis of the IGS MEGX data shows that there are no obvious changes related to the elevation angle in the MP observations of QZSS, which is independent of the multipath environment and its surrounding area and more inclined to come from the satellite itself.

4.4.2. MP Observations of GEO Satellites. MP observations of GEO satellites are more complex than that of IGSO; the GEO satellites of the Satellite-Based Augmentation System (SBAS) also emit $L 1$ and $L 5$ dual-frequency signals. The MP change law of the SBAS system is different from that of Beidou GEO satellites. MP observations of SBAS S127 collected at CUT0, JFNG, and MAYG stations with an elevation of $16^{\circ}, 17^{\circ}$, and $71^{\circ}$, respectively, are shown in Figure 8.

The MP values after the 2500 epoch in Figure 8 change strongly for both $L 1$ and $L 5$, and the fluctuation period of $L 5$ is 2 hours at the same time in the receiver of the three different stations, which is caused mainly by the satellite signals. The fluctuation of $L 1$ decreases with the increase of the elevation angle.

\section{Suppression of MP Signals}

5.1. Pseudorange Correction Model Based on Elevation. MP observations of Beidou satellites show a change related to elevation, which is called the code-carrier divergence phenomenon [21]. In Section 4, a mathematical model of pseudorange multipath error is established, which can be applied to MEO, IGSO, and GEO satellites. But it does not consider the code-carrier divergence phenomenon of the Beidou satellite, so a statistical model based on elevation can be used to describe the code and carrier divergence of Beidou IGSO and MEO satellites. Instead of considering the cause of code and carrier divergence, the model only repairs it statistically. The GPS SVN49 satellite launched in March 2009 also showed a code and carrier divergence; different from that of Beidou, the code and carrier divergence of the $L 1$ frequency of SVN49 is not obvious when the elevation is less than $40^{\circ}$; otherwise, it increases with the elevation; however, the code and carrier divergence of $L 2$ frequency will decrease first and then increase when the elevation exceeds $40^{\circ}$. Hauschild et al. [22] collected the orthogonal signal of GPS SVN49 with a high gain antenna, separated the delay, relative amplitude, and phase difference of the multipath signal on $L 1$ and $L 2$ by impulse response and chip shape, explained the origin of the multipath by the GPS satellite antenna parameters and radiofrequency circuit structure, and, finally, reproduced the MP fluctuation and code-carrier divergence with these parameters.

Using the above model, the simulation results of MP fluctuation of the GEO satellite are illustrated (see Figure 9).

Based on the statistical analysis of the measured MP, an elevation-based pseudorange correction model is established with $B 1, B 2$, and $B 3$, three frequencies, using the continuous observations for several cycles, which is shown in detail (see Table 1).

Because of the ambiguity of carrier phase observation, the MP value is a relative quantity, and its average value is artificially set to zero. Therefore, the absolute value of the pseudorange correction cannot be obtained when the elevation pseudorange correction model is established based on the MP observations. That is, if a constant is added to the pseudorange correction $\Delta p$ in the model, the modified MP value remains unchanged, but the location results may change.

5.2. Sidereal Filtering Based on Satellite Elevation. Usually, the multipath error period of the GEO satellite is between 86184 and 86201 seconds, which is very close to a stellar day period (86164 seconds). Therefore, to weaken the influence of multipath error on positioning, a sidereal filtering is often used to correct the pseudorange observation. 

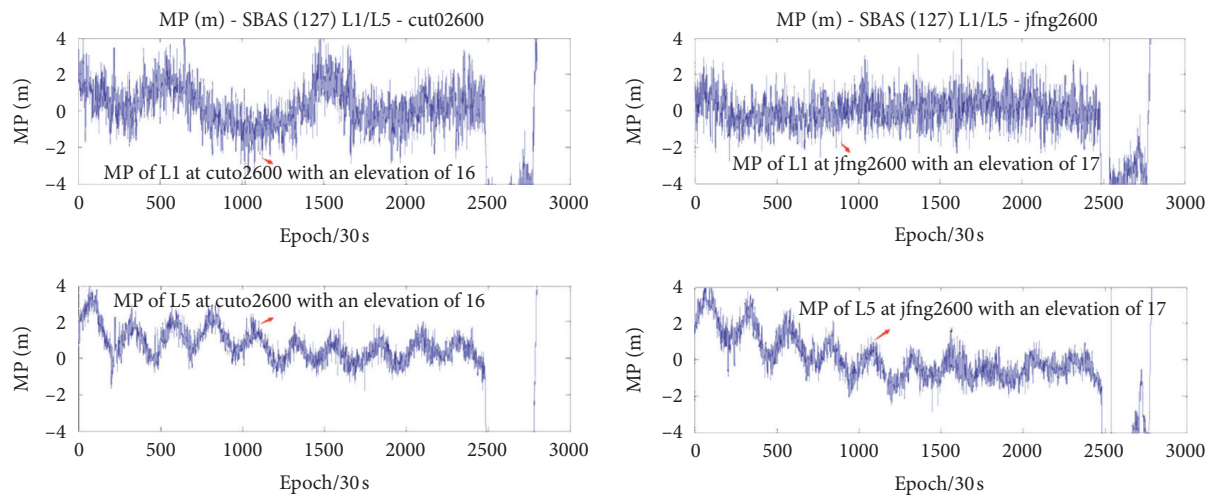

(a)

(b)
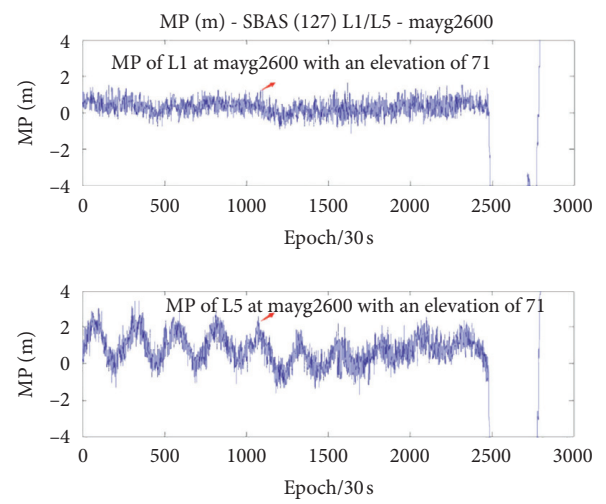

(c)

FIgURE 8: MP observations of SBAS S127. (a) CUT0 Station. (b) JFNG Station. (c) MAYG Station.

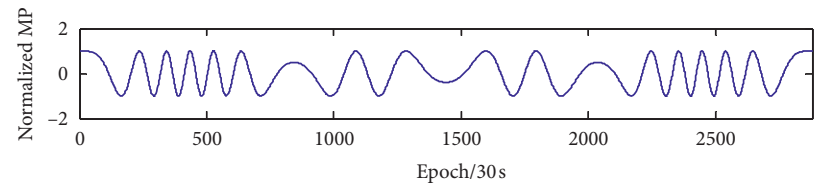

(a)

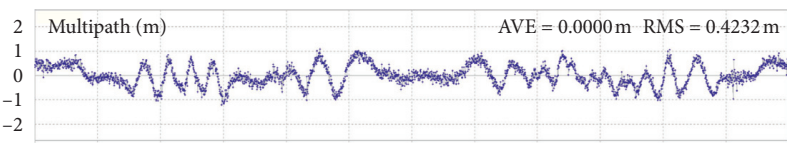

(b)

Figure 9: MP observations of the GEO satellite. (a) Simulation data. (b) Measured data.

TABLE 1: Pseudorange correction $\Delta p(\mathrm{~m})[21]$.

\begin{tabular}{|c|c|c|c|c|c|c|}
\hline Elevation (deg) & B1: MEO & B2: MEO & B3: MEO & B1: IGSO & B2: IGSO & B3: IGSO \\
\hline 0 & -0.47 & -0.40 & -0.22 & -0.55 & -0.71 & -0.27 \\
\hline 10 & -0.38 & -0.31 & -0.15 & -0.40 & -0.36 & -0.23 \\
\hline 20 & -0.32 & -0.26 & -0.13 & -0.34 & -0.33 & -0.21 \\
\hline 30 & -0.23 & -0.18 & -0.10 & -0.23 & -0.19 & -0.15 \\
\hline 40 & -0.11 & -0.06 & -0.04 & -0.15 & -0.14 & -0.11 \\
\hline 50 & 0.06 & 0.09 & 0.05 & -0.04 & -0.03 & -0.04 \\
\hline 60 & 0.34 & 0.28 & 0.14 & 0.09 & 0.08 & 0.05 \\
\hline 70 & 0.69 & 0.48 & 0.27 & 0.19 & 0.17 & 0.14 \\
\hline 80 & 0.97 & 0.64 & 0.36 & 0.27 & 0.24 & 0.19 \\
\hline 90 & 1.05 & 0.69 & 0.47 & 0.35 & 0.33 & 0.32 \\
\hline
\end{tabular}

The main principle of the sidereal filtering is to use the strong correlation of the two consecutive days' data to correct the multipath error. Usually, the data features of the first day are extracted to correct the data for the second day. Due to the difference of 236 seconds between a stellar day and a solar day, it is necessary to translate the previous day's data when using a sidereal filtering [23]. The principle of the sidereal filtering is shown in Figure 10.

The pseudorange MP fluctuation of Beidou GEO and IGSO satellites can be alleviated with a sidereal filtering. In 


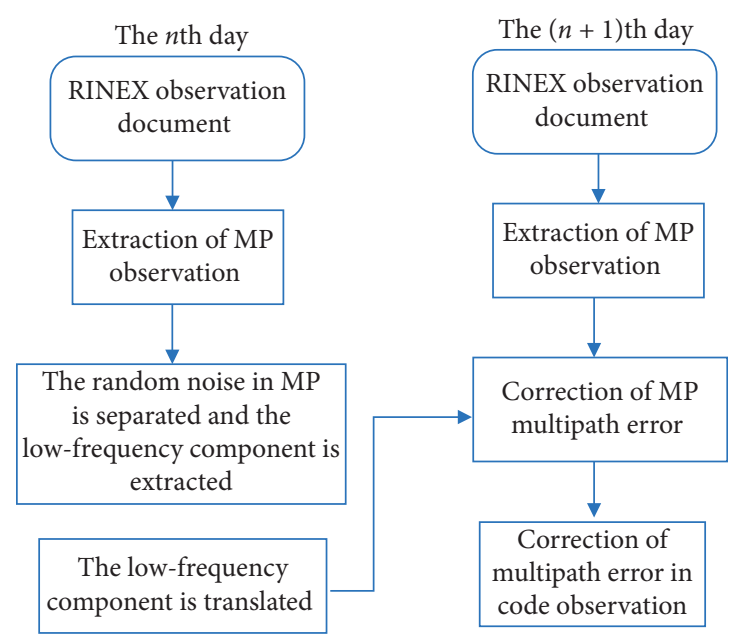

FIGURE 10: Working principle of the sidereal filtering.

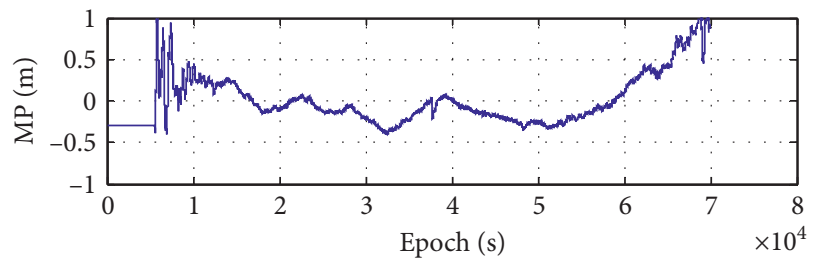

(a)

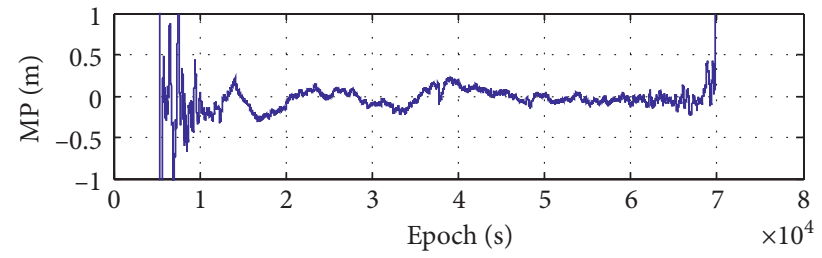

(b)

FIGURE 11: MP observations before and after the correction of sidereal filtering. (a) Original MP observations. (b) Revised MP observations after sidereal filtering.

the elevation-based pseudorange correction model, the mean value of the all-day pseudorange measurement must remain unchanged; the pseudorange measurement noises of the two consecutive days are independent, even if the lowpass filter has filtered out the high-frequency component of the noise; the cost of the sidereal filtering is the increase of the measurement noise.

Data of Beidou C06 collected with the Trimble BD930 receiver on October 7th and 8th in 2018 is used for analysis; the MP observations before and after the correction of sidereal filtering are shown in Figure 11, where the MP observations range within $(-0.4,1) \mathrm{m}$ before sidereal filtering, but after sidereal filtering, the MP observations range within $(-0.3,0.2) \mathrm{m}$ with a fluctuation range reduction of $35.7 \%$ when the receiver reaches a steady state.

\section{Conclusions}

In GNSS systems, satellite multipath interference is a nonnegligible factor restricting the overall performance of the satellite navigation systems. Based on the ground multipath reflection model, it deeply studies the influence of GNSS satellite orbit parameters on multipath fading frequency and establishes a multipath signal model related to satellite orbit parameters. Through collecting the measured data of different stations and satellites, the influence of carrier phase cycle slip, receiver clock adjustment, and GNSS satellite orbit on MP observation is analyzed in detail. The statistical analysis of the measured data shows that the multipath fading characteristics of the IGSO satellite and the MEO satellite are related to the satellite elevation, and there is a code-carrier divergence phenomenon, which is not obvious in the GEO satellite. Finally, aiming at the common phenomenon of code-carrier divergence in the Beidou system, the elevation-based pseudorange correction model and a sidereal filtering are built to correct the MP errors, and finally, the measured data are used to verify the model. The MP model built in this paper explains the source of the periodic fluctuation of the MP value but does not explain the phenomenon of Beidou satellite codecarrier divergence. Also, the elevation model and sidereal filtering can be used to repair code-carrier divergence in precise single point positioning, but its practical application is very limited. So it is necessary to study the cause of code-carrier divergence and establish a widely used MP model in the future.

\section{Data Availability}

The data used to support the findings of this study are included within the article.

\section{Conflicts of Interest}

The authors declare that there are no conflicts of interest regarding the publication of this paper. 


\section{Acknowledgments}

This work was supported in part by the National Major Fundamental Research Program of China (Grant no. GFZX0301040115), National Natural Science Foundation of China (Grant no. 61571188), and Construct Program of the Key Discipline in Hunan Province.

\section{References}

[1] Y. Liu, B. Lian, and T. Zhou, "Gaussian message passing-based cooperative localization with node selection scheme in wireless networks," Signal Processing, vol. 156, pp. 166-176, 2019.

[2] A. Kumar and A. K. Singh, "A novel multipath mitigation technique for GNSS signals in urban scenarios," IEEE Transactions on Vehicular Technology, vol. 69, no. 3, pp. 2649-2658, 2020.

[3] T. Y. Zhou, Y. Cheng, and B. W. Lian, "Research on highprecision passive localization based on phase difference changing rate," Concurrency and Computation Practice \& Experience, vol. 31, no. 23, Article ID e4492, 2019.

[4] C. Chen, G. Chang, N. Zheng, and T. Xu, "GNSS multipath error modeling and mitigation by using sparsity-promoting regularization," IEEE Access, vol. 7, pp. 24096-24108, 2019.

[5] T. Y. Zhou, B. W. Lian, S. Q. Yang et al., "Improved GNSS cooperation positioning algorithm for indoor localization," Computer, Materials \&Continue, vol. 56, no. 2, pp. 225-245, 2018.

[6] C. H. Chen, F. Y. Song, F. J. Hwang et al., "A probability density function generator based on neural networks," Physica A: Statistical Mechanics and Its Applications, vol. 541, Article ID 123344, 2020.

[7] Y. Y. Liu, B. W. Lian, and T. Y. Zhou, "Gaussian message passing-based cooperative localization with node selection scheme in wireless networks," Signal Processing, vol. 156, pp. 166-176, 2018.

[8] T. Y. Zhou, B. W. Lian, D. D. Yang et al., "Research on GPS geometry-based observational stochastic error model," Journal on Communications, vol. 40, no. 9, pp. 1-12, 2019.

[9] Y. Wang, Y. Wu, and Y. Shen, "Joint spatiotemporal multipath mitigation in large-scale Array localization," IEEE Transactions on Signal Processing, vol. 67, no. 3, pp. 783-797, 2019.

[10] P. Wu, B. Lian, Y. Song, and Z. Yue, "Study on multipath effect of GEO satellite in bei dou navigation satellite system," in Proceedings of the China Satellite Navigation Conference (CSNC) 2016 Proceedings, pp. 347-358, Singapore, April 2016.

[11] H. Ko, B. Kim, and S.-H. Kong, "GNSS multipath-resistant cooperative navigation in urban vehicular networks," IEEE Transactions on Vehicular Technology, vol. 64, no. 12, pp. 5450-5463, 2015.

[12] C.-H. Chen, "A cell probe-based method for vehicle speed estimation," IEICE Transactions on Fundamentals of Electronics, Communications and Computer Sciences, vol. 103, no. 1, pp. 265-267, 2020.

[13] D. Li, P. Zhang, J. Zhao et al., "MP mitigation in GNSS positioning by GRU neural networks and adaptive wavelet filtering," IET Communications, vol. 13, no. 17, pp. 1-10, 2019.

[14] W. Mu, Z. Wang, L. Guo, and Y. Liu, "A framework of multipath mitigation with joint multipath-Doppler diversity for RF relative measurement," Institute of Electrical and Electronics Engineer Access, vol. 8, pp. 166839-166851, 2020.
[15] J. Park, S. V. Veettil, M. Aquino, L. Yang, and C. Cesaroni, "Mitigation of ionospheric effects on GNSS positioning at low latitudes," Navigation, vol. 64, no. 1, pp. 67-74, 2017.

[16] G. Hu, L. Ni, B. Gao, X. Zhu, W. Wang, and Y. Zhong, "Model predictive based unscented kalman filter for hypersonic vehicle navigation with INS/GNSS integration," Institute of Electrical and Electronics Engineer Access, vol. 8, pp. 48144823, 2020.

[17] Y. Tao, C. Liu, T. Chen et al., "Real-time multipath mitigation in multi-GNSS short baseline positioning via CNN-LSTM method," Mathematical Problems in Engineering, vol. 2021, Article ID 6573230, 12 pages, 2021.

[18] J. Chang, X. Zhan, Y. Zhai, S. Wang, and K. Lin, "Analysis of BDS GEO satellite multipath effect for GNSS integrity monitoring in civil aviation," Aerospace Systems, vol. 23, 2021.

[19] M. M. Caballú, A. L. Swindlehurst, and G. S. Granadosa, "Power-based capon beamforming: avoiding the cancellation effects of GNSS multipat," Signal Processing, vol. 180, pp. 1-9, 2020.

[20] L. Wanninger and S. Beer, "BeiDou satellite-induced code pseudorange variations: diagnosis and therapy," GPS Solutions, vol. 19, no. 4, pp. 639-648, 2015.

[21] B. W. Parkinson, Multipath Effects Global Positioning SystemAmerican Institute of Aeronautics and Astronautics, Washington, DC, USA, 1996.

[22] A. Hauschild, O. Montenbruck, S. Thoelert, S. Erker, M. Meurer, and J. Ashjaee, "A multi-technique approach for characterizing the SVN49 signal anomaly, part 1: receiver tracking and IQ constellation," GPS Solutions, vol. 16, no. 1, pp. 19-28, 2012.

[23] S. Thoelert, M. Meurer, S. Erker, O. Montenbruck, A. Hauschild, and P. Fenton, "A multi-technique approach for characterizing the SVN49 signal anomaly, part 2: chip shape analysis," GPS Solutions, vol. 16, no. 1, pp. 29-39, 2012. 